\title{
Compact operators on some Fibonacci difference sequence spaces
}

\author{
Abdullah Alotaibi ${ }^{1}$, Mohammad Mursaleen ${ }^{1,2^{*}}$, Badriah AS Alamri $^{1}$ and Syed Abdul Mohiuddine ${ }^{1}$
}

"Correspondence:

mursaleenm@gmail.com

${ }^{1}$ Operator Theory and Applications Research Group, Department of Mathematics, Faculty of Science, King Abdulaziz University, P.O. Box 80203, Jeddah, 21589, Saudi Arabia ${ }^{2}$ Department of Mathematics, Aligarh Muslim University, Aligarh, 202 002, India

\begin{abstract}
In this paper, we characterize the matrix classes $\left(\ell_{1}, \ell_{p}(\widehat{F})\right)(1 \leq p<\infty)$, where $\ell_{p}(\widehat{F})$ is some Fibonacci difference sequence spaces. We also obtain estimates for the norms of the bounded linear operators $L_{A}$ defined by these matrix transformations and find conditions to obtain the corresponding subclasses of compact matrix operators by using the Hausdorff measure of noncompactness.
\end{abstract}

MSC: 46A45; 11B39; 46B50

Keywords: sequence spaces; Fibonacci numbers; compact operators; Hausdorff measure of noncompactness

\section{Introduction and preliminaries}

Let $\mathbb{N}=\{0,1,2, \ldots\}$ and $\mathbb{R}$ be the set of all real numbers. We shall write $\lim _{k}, \sup _{k}$, inf $k$, and $\sum_{k}$ instead of $\lim _{k \rightarrow \infty}, \sup _{k \in \mathbb{N}}, \inf _{k \in \mathbb{N}}$, and $\sum_{k=0}^{\infty}$, respectively. Let $\omega$ be the vector space of all real sequences $x=\left(x_{k}\right)_{k \in \mathbb{N}}$. By the term sequence space, we shall mean any linear subspace of $\omega$. Let $\varphi, \ell_{\infty}, c$, and $c_{0}$ denote the sets of all finite, bounded, convergent and null sequences, respectively. We write $\ell_{p}=\left\{x \in \omega: \sum_{k}\left|x_{k}\right|^{p}<\infty\right\}$ for $1 \leq p<\infty$. Also, we shall use the conventions that $e=(1,1, \ldots)$ and $e^{(n)}$ is the sequence whose only non-zero term is 1 in the $n$th place for each $n \in \mathbb{N}$. For any sequence $x=\left(x_{k}\right)$, let $x^{[n]}=\sum_{k=0}^{n} x_{k} e^{(k)}$ be its $n$-section. Moreover, we write $b s$ and $c s$ for the sets of sequences with bounded and convergent partial sums, respectively.

A $B$-space is a complete normed space. A topological sequence space in which all coordinate functionals $\pi_{k}, \pi_{k}(x)=x_{k}$, are continuous is called a $K$-space. A $B K$-space is defined as a $K$-space which is also a $B$-space, that is, a $B K$-space is a Banach space with continuous coordinates. A $B K$-space $X \supset \varphi$ is said to have $A K$ if every sequence $x=\left(x_{k}\right) \in X$ has a unique representation $x=\sum_{k} x_{k} e^{(k)}$. For example, the space $\ell_{p}(1 \leq p<\infty)$ is $B K$-space with $\|x\|_{p}=\left(\sum_{k}\left|x_{k}\right|^{p}\right)^{1 / p}$ and $c_{0}, c$, and $\ell_{\infty}$ are $B K$-spaces with $\|x\|_{\infty}=\sup _{k}\left|x_{k}\right|$. Further, the $B K$-spaces $c_{0}$ and $\ell_{p}$ have $A K$, where $1 \leq p<\infty$ (cf. [1]).

A sequence $\left(b_{n}\right)$ in a normed space $X$ is called a Schauder basis for $X$ if for every $x \in X$ there is a unique sequence $\left(\alpha_{n}\right)$ of scalars such that $x=\sum_{n} \alpha_{n} b_{n}$, i.e., $\lim _{m} \| x-$ $\sum_{n=0}^{m} \alpha_{n} b_{n} \|=0$.

The $\beta$-dual of a sequence space $X$ is defined by

$$
X^{\beta}=\left\{a=\left(a_{k}\right) \in \omega: a x=\left(a_{k} x_{k}\right) \in c s \text { for all } x=\left(x_{k}\right) \in X\right\} .
$$

(c) 2015 Alotaibi et al. This article is distributed under the terms of the Creative Commons Attribution 4.0 International License (http://creativecommons.org/licenses/by/4.0/), which permits unrestricted use, distribution, and reproduction in any medium, provided you give appropriate credit to the original author(s) and the source, provide a link to the Creative Commons license, and indicate if changes were made. 
Let $A=\left(a_{n k}\right)_{n, k=0}^{\infty}$ be an infinite matrix of real numbers $a_{n k}$, where $n, k \in \mathbb{N}$. We write $A_{n}$ for the sequence in the $n$th row of $A$, that is, $A_{n}=\left(a_{n k}\right)_{k=0}^{\infty}$ for every $n \in \mathbb{N}$. In addition, if $x=\left(x_{k}\right)_{k=0}^{\infty} \in \omega$ then we define the $A$-transform of $x$ as the sequence $A x=\left\{A_{n}(x)\right\}_{n=0}^{\infty}$, where

$$
A_{n}(x)=\sum_{k=0}^{\infty} a_{n k} x_{k} \quad(n \in \mathbb{N})
$$

provided the series on the right side converges for each $n \in \mathbb{N}$.

For arbitrary subsets $X$ and $Y$ of $\omega$, we write $(X, Y)$ for the class of all infinite matrices that map $X$ into $Y$. Thus, $A \in(X, Y)$ if and only if $A_{n} \in X^{\beta}$ for all $n \in \mathbb{N}$ and $A x \in Y$ for all $x \in X$.

The matrix domain $X_{A}$ of an infinite matrix $A$ in sequence space $X$ is defined by

$$
X_{A}=\left\{x=\left(x_{k}\right) \in \omega: A x \in X\right\}
$$

which is a sequence space.

The following results are very important in our study $[2,3]$.

Lemma 1.1 Let $X \supset \phi$ and $Y$ be $B K$-spaces.

(a) Then we have $(X, Y) \subset \mathcal{B}(X, Y)$, that is, every matrix $A \in(X, Y)$ defines an operator $L_{A} \in \mathcal{B}(X, Y)$ by $L_{A}(x)=A x$ for all $x \in X$.

(b) If $X$ has $A K$, then $\mathcal{B}(X, Y) \subset(X, Y)$, that is, for every operator $L \in \mathcal{B}(X, Y)$ there exists a matrix $A \in(X, Y)$ such that $L(x)=A x$ for all $x \in X$.

The Fibonacci numbers are the sequence of numbers $\left\{f_{n}\right\}_{n=0}^{\infty}$ defined by the linear recurrence equations

$$
f_{0}=f_{1}=1 \quad \text { and } \quad f_{n}=f_{n-1}+f_{n-2} ; \quad n \geq 2 .
$$

Fibonacci numbers have many interesting properties and applications in arts, sciences and architecture. For example, the ratio sequences of Fibonacci numbers converge to the golden ratio which is important in sciences and arts. Also, some basic properties of Fibonacci numbers can be found in [4].

Throughout, let $1 \leq p \leq \infty$ and $q$ denote the conjugate of $p$, that is, $q=p /(p-1)$ for $1<p<\infty, q=\infty$ for $p=1$ or $q=1$ for $p=\infty$.

Recently, in [5] and [6], the Fibonacci difference sequence spaces $\ell_{p}(\widehat{F})$ and $\ell_{\infty}(\widehat{F})$ have been defined as follows:

$$
\ell_{p}(\widehat{F})=\left\{x=\left(x_{n}\right) \in \omega: \sum_{n}\left|\frac{f_{n}}{f_{n+1}} x_{n}-\frac{f_{n+1}}{f_{n}} x_{n-1}\right|^{p}<\infty\right\} ; \quad 1 \leq p<\infty
$$

and

$$
\ell_{\infty}(\widehat{F})=\left\{x=\left(x_{n}\right) \in \omega: \sup _{n \in \mathbb{N}}\left|\frac{f_{n}}{f_{n+1}} x_{n}-\frac{f_{n+1}}{f_{n}} x_{n-1}\right|<\infty\right\}
$$


Using the notation of the matrix domain, the sequence spaces $\ell_{p}(\widehat{F})$ and $\ell_{\infty}(\widehat{F})$ may be redefined by

$$
\ell_{p}(\widehat{F})=\left(\ell_{p}\right)_{\widehat{F}} \quad(1 \leq p<\infty) \quad \text { and } \quad \ell_{\infty}(\widehat{F})=\left(\ell_{\infty}\right)_{\widehat{F}}
$$

where the matrix $\widehat{F}=\left(\widehat{f}_{n k}\right)$ is defined by

$$
\widehat{f}_{n k}=\left\{\begin{array}{ll}
-\frac{f_{n+1}}{f_{n}} & (k=n-1), \\
\frac{f_{n}}{f_{n+1}} & (k=n), \\
0 & (0 \leq k<n-1) \text { or }(k>n)
\end{array} \quad(n, k \in \mathbb{N})\right.
$$

Further, it is clear that the spaces $\ell_{p}(\widehat{F})$ and $\ell_{\infty}(\widehat{F})$ are $B K$ spaces with the norms given by

$$
\|x\|_{\ell_{p}(\widehat{F})}=\left(\sum_{n}\left|y_{n}(x)\right|^{p}\right)^{1 / p} ; \quad(1 \leq p<\infty) \text { and }\|x\|_{\ell_{\infty}(\widehat{F})}=\sup _{n \in \mathbb{N}}\left|y_{n}(x)\right| \text {, }
$$

where the sequence $y=\left(y_{n}\right)=\left(\widehat{F}_{n}(x)\right)$ is the $\widehat{F}$-transform of a sequence $x=\left(x_{n}\right)$, i.e.,

$$
y_{n}=\widehat{F}_{n}(x)=\left\{\begin{array}{ll}
\frac{f_{0}}{f_{1}} x_{0}=x_{0} & (n=0), \\
\frac{f_{n}}{f_{n+1}} x_{n}-\frac{f_{n+1}}{f_{n}} x_{n-1} & (n \geq 1)
\end{array} \quad(n \in \mathbb{N}) .\right.
$$

\section{Methods of measure of noncompactness}

The first measure of noncompactness, the function $\alpha$, was defined and studied by Kuratowski [7] in 1930. Darbo [8] used this measure to generalize both the classical Schauder fixed point principle and (a special variant of) the Banach contraction mapping principle for so-called condensing operators. The Hausdorff or ball measure of noncompactness $\chi$ was introduced by Goldenštein et al. [9] in 1957, and later studied by Goldenštein and Markus [10].

Let $X$ and $Y$ be infinite dimensional Banach spaces. We recall that a linear operator $L$ from $X$ into $Y$ is called compact if its domain is all of $X$ and, for every bounded sequence $\left(x_{n}\right)$ in $X$, the sequence the sequence $\left(L\left(x_{n}\right)\right)$ has a convergent subsequence. We denote the class of all compact operators in $\mathcal{B}(X, Y)$ by $\mathcal{C}(X, Y)$.

Let $(X, d)$ be a metric space, $x_{0} \in X$ and $r>0$. Then we write, as usual, $B(x, r)=\{x \in$ $\left.X: d\left(x, x_{0}\right)<r\right\}$ for the open ball of radius $r$ and center $x_{0}$. Let $\mathcal{M}_{X}$ denote the class of all bounded subsets of $X$. If $Q \in \mathcal{M}_{X}$, then the Hausdorff measure of noncompactness of the set $Q$, denoted by $\chi(Q)$, is defined by

$$
\chi(Q)=\inf \left\{\epsilon>0: Q \subset \bigcup_{k=1}^{n} B\left(x_{k}, r_{k}\right), x_{k} \in X, r_{k}<\epsilon(k=1,2, \ldots), n \in \mathbb{N}\right\} .
$$

The function $\chi: \mathcal{M}_{X} \rightarrow[0, \infty)$ is called the Hausdorff measure of noncompactness.

The basic properties of the Hausdorff measure of noncompactness can be found in [3, $11-14]$.

Now we recall the definition of the Hausdorff measure of noncompactness operators between Banach spaces. Let $X$ and $Y$ be Banach spaces and $\chi_{1}$ and $\chi_{2}$ be the Hausdorff 
measures of noncompactness on $X$ and $Y$, respectively. An operator $L: X \rightarrow Y$ is said to be $\left(\chi_{1}, \chi_{2}\right)$-bounded if $L(Q) \in \mathcal{M}_{Y}$ for all $Q \in \mathcal{M}_{X}$ and there exists a constant $C \geq 0$ such that $\chi_{2}(L(Q)) \leq C \chi_{1}(Q)$ for all $Q \in \mathcal{M}_{X}$. If an operator $L$ is $\left(\chi_{1}, \chi_{2}\right)$-bounded then the number

$$
\|L\|_{\left(\chi_{1}, \chi_{2}\right)}=\inf \left\{C \geq 0: \chi_{2}(L(Q)) \leq C \chi_{1}(Q) \text { for all } Q \in \mathcal{M}_{X}\right\}
$$

is called the $\left(\chi_{1}, \chi_{2}\right)$-measure of noncompactness of $L$. If $\chi_{1}=\chi_{2}=\chi$, then we write $\|L\|_{\left(\chi_{1}, \chi_{2}\right)}=\|L\|_{\chi}$.

Now we outline the applications of the Hausdorff measure of noncompactness to the characterization of compact operators between Banach spaces. Let $X$ and $Y$ be Banach spaces and $L \in \mathcal{B}(X, Y)$. Then the Hausdorff measure of noncompactness of $L$ is given by ([14], Theorem 2.25)

$$
\|L\|_{\chi}=\chi\left(L\left(S_{X}\right)\right)
$$

and $L$ is compact if and only if ([14], Corollary $2.26(2.58))$

$$
\|L\|_{\chi}=0 .
$$

The identities in (2.1) and (2.2) reduce the characterization of compact operators $L \in$ $\mathcal{B}(X, Y)$ to the determination of the Hausdorff measure of noncompactness $\chi(Q)$ of bounded sets $Q$ in a Banach space $X$. If $X$ has a Schauder basis, then there exist estimates or even identities for $\chi(Q)$.

Theorem 2.1 ([9] or [14], Theorem 2.23) Let X be a Banach space with a Schauder basis $\left(b_{k}\right)_{k=0}^{\infty}, Q \in \mathcal{M}_{X}, \mathcal{P}_{n}: X \rightarrow X$ be the projectors onto the linear span of $\left\{b_{0}, b_{1}, \ldots, b_{n}\right\}$ and $\mathcal{R}_{n}=I-\mathcal{P}_{n}$ for $n=0,1, \ldots$, where I denotes the identity map on $X$. Then we have

$$
\frac{1}{a} \cdot \limsup \left(\sup _{x \in Q}\left\|\mathcal{R}_{n}(x)\right\|\right) \leq \chi(Q) \leq \limsup _{n \rightarrow \infty}\left(\sup _{x \in Q}\left\|\mathcal{R}_{n}(x)\right\|\right)
$$

where $a=\limsup _{n \rightarrow \infty}\left\|\mathcal{R}_{n}\right\|$.

In particular, the following result shows how to compute the Hausdorff measure of noncompactness in the spaces $c_{0}$ and $\ell_{p}(1 \leq p<\infty)$, which are $B K$-spaces with $A K$.

Theorem 2.2 ([14], Theorem 2.15) Let $Q$ be a bounded subset of the normed space $X$, where $X$ is $\ell_{p}$ for $1 \leq p<\infty$ or $c_{0}$. If $P_{n}: X \rightarrow X$ is the operator defined by $\mathcal{P}_{n}(x)=x^{[n]}$ for all $x=\left(x_{k}\right)_{k=0}^{\infty} \in X$ and $\mathcal{R}_{n}=I-\mathcal{P}_{n}$ for $n=0,1, \ldots$, then we have

$$
\chi(Q)=\lim _{n \rightarrow \infty}\left(\sup _{x \in Q}\left\|\mathcal{R}_{n}(x)\right\|\right)
$$

Since matrix mappings between $B K$ spaces define bounded linear operators between these spaces which are Banach spaces, it is natural to use the above results and the Hausdorff measure of noncompactness to obtain necessary and sufficient conditions for matrix operators between $B K$ spaces with a Schauder basis or $A K$ to be compact operators. This 
technique has recently been used by several authors in many research papers (see for instance [15-25]). In this paper, we characterize the matrix classes $\left(\ell_{1}, \ell_{p}(\widehat{F})\right)(1 \leq p<\infty)$ and obtain an identity for the norms of the bounded linear operators $L_{A}$ defined by these matrix transformations. We also find conditions to obtain the corresponding subclasses of compact matrix operators by using the Hausdorff measure of noncompactness.

\section{Main results}

In [26], the classes $\left(\ell_{p}(\widehat{F}), Y\right),\left(\ell_{\infty}(\widehat{F}), Y\right),\left(\ell_{1}(\widehat{F}), Y\right), Y=\ell_{\infty}, c_{0}, c$, and $\left(\ell_{p}(\widehat{F}), \ell_{1}\right),\left(\ell_{1}(\widehat{F}), \ell_{p}\right)$ of compact operators were characterized. In this paper, we characterize the classes $\mathcal{B}\left(\ell_{1}, \ell_{p}^{\lambda}\right)$ for $(1 \leq p<\infty)$ and compute the norm of operators in $\mathcal{B}\left(\ell_{1}, \ell_{p}^{\lambda}\right)$. We also apply the results of the previous section to determine the Hausdorff measure of noncompactness of operators in $\mathcal{B}\left(\ell_{1}, \ell_{p}^{\lambda}\right)$, and to characterize the classes $\mathcal{C}\left(\ell_{1}, \ell_{p}\right)$ for $1 \leq p<\infty$.

Here we characterize the classes $\mathcal{B}\left(\ell_{1}, \ell_{p}(\widehat{F})\right)$ for $(1 \leq p<\infty)$ and compute the norm of operators in $\mathcal{B}\left(\ell_{1}, \ell_{p}(\widehat{F})\right)$. We also apply the results of the previous section to determine the Hausdorff measure of noncompactness of operators in $\mathcal{B}\left(\ell_{1}, \ell_{p}(\widehat{F})\right)$ and to characterize the classes $\mathcal{C}\left(\ell_{1}, \ell_{p}(\widehat{F})\right)$ for $1 \leq p<\infty$.

The following result is useful.

Lemma 3.1 ([14], Theorem 3.8) Let $T$ be a triangle and $X$ and $Y$ be arbitrary subsets of $\omega$.

(a) Then we have $A \in\left(X, Y_{T}\right)$ if and only if $C=T \cdot A \in(X, Y)$, where $C$ denotes the matrix product of $T$ and $A$.

(b) If $X$ and $Y$ are $B$ spaces and $A \in\left(X, Y_{T}\right)$ then

$$
\left\|L_{A}\right\|=\left\|L_{C}\right\|
$$

First we establish the characterizations of the classes $\mathcal{B}\left(\ell_{1}, \ell_{p}(\widehat{F})\right)$ for $(1 \leq p<\infty)$ and an identity for the operator norm.

Theorem 3.1 Let $1 \leq p<\infty$.

(a) We have $L \in \mathcal{B}\left(\ell_{1}, \ell_{p}(\widehat{F})\right)$ if and only if there exists an infinite matrix $A \in\left(\ell_{1}, \ell_{p}(\widehat{F})\right)$ such that

$$
\|A\|=\sup _{k}\left(\sum_{n}\left|\frac{f_{n}}{f_{n+1}} a_{n k}-\frac{f_{n+1}}{f_{n}} a_{n-1, k}\right|^{p}\right)^{1 / p}<\infty
$$

and

$$
L(x)=A x \quad \text { for all } x \in \ell_{1} .
$$

(b) If $L \in \mathcal{B}\left(\ell_{1}, \ell_{p}(\widehat{F})\right)$ then

$$
\|L\|=\|A\| .
$$

Proof Since $\ell_{1}$ is a $B K$ space with $A K$ it follows from Lemma 1.1 that $L \in \mathcal{B}\left(\ell_{1}, \ell_{p}(\widehat{F})\right)$ for $1 \leq p<\infty$ if and only if there exists an infinite matrix $A \in\left(\ell_{1}, \ell_{p}(\widehat{F})\right)$ such that (3.3) holds. 
Also we have by Lemma 3.1(a) that $A \in\left(\ell_{1}, \ell_{p}(\widehat{F})\right)$ if and only if $C=\widehat{F} \cdot A \in\left(\ell_{1}, \ell_{p}\right)$, where the matrix $C=\left(c_{n k}\right)$ is defined by

$$
c_{n k}=\frac{f_{n}}{f_{n+1}} a_{n k}-\frac{f_{n+1}}{f_{n}} a_{n-1, k} .
$$

Furthermore, we have by [2], Example 8.4.1D, $C \in\left(\ell_{1}, \ell_{p}\right)$ if and only if

$$
\|C\|=\sup _{k}\left(\sum_{n=0}^{\infty}\left|c_{n k}\right|^{p}\right)^{1 / p}<\infty
$$

This completes the proof of part (a).

(b) If $L \in \mathcal{B}\left(\ell_{1}, \ell_{p}^{\lambda}\right)$ then it follows from (3.1) that $\|L\|=\left\|L_{C}\right\|$, where $L_{C} \in \mathcal{B}\left(\ell_{1}, \ell_{p}\right)$ is given by $L_{C}(x)=C x$ for all $x \in \ell_{1}$. It follows by the Minkowski inequality that

$$
\begin{aligned}
\left\|L_{C}(x)\right\|_{p} & =\left(\sum_{n=0}^{\infty}\left|\sum_{k=0}^{\infty} c_{n k} x_{k}\right|^{p}\right)^{1 / p} \leq \sum_{k=0}^{\infty}\left|x_{k}\right|\left(\sum_{n=0}^{\infty}\left|c_{n k}\right|^{p}\right)^{1 / p} \\
& \leq\|C\| \cdot\|x\|=\|A\| \cdot\|x\|,
\end{aligned}
$$

and so

$$
\|L\| \leq\|A\|
$$

We also obtain for $e^{(k)} \in S_{\ell_{1}}(k \in \mathbb{N})$

$$
\left\|L_{C}\left(e^{(k)}\right)\right\|=\left(\sum_{n=0}^{\infty}\left|c_{n k}\right|^{p}\right)^{p}
$$

and so $\|L\| \geq\|A\|$. This and (3.5) yield (3.4).

This completes the proof.

Now we are going to establish a formula for the Hausdorff measure of noncompactness of operators in $\mathcal{B}\left(\ell_{1}, \ell_{p}(\widehat{F})\right)$. We need the following result.

Lemma $3.2([1]$, Theorem 4.2) Let $X$ be a linear metric space with a translation invariant metric, $T$ be a triangle and $\chi$, and $\chi_{T}$ denote the Hausdorff measures of noncompactness on $\mathcal{M}_{X}$ and $\mathcal{M}_{X_{T}}$, respectively. Then $\chi_{T}(Q)=\chi(T Q)$ for all $Q \in \mathcal{M}_{X_{T}}$.

Theorem 3.2 Let $L \in \mathcal{B}\left(\ell_{1}, \ell_{p}(\widehat{F})\right)(1 \leq p<\infty)$ and A denote the matrix which represents $L$. Then we have

$$
\|L\|_{\chi_{\ell p}(\widehat{F})}=\lim _{m \rightarrow \infty}\left(\sup _{k} \sum_{n=m}^{\infty}\left|\frac{f_{n}}{f_{n+1}} a_{j k}-\frac{f_{n+1}}{f_{n}} a_{j-1, k}\right|^{p}\right)^{1 / p} .
$$

Proof We write $S=S_{\ell_{1}}$, for short, and $C^{[m]}(m \in \mathbb{N})$ for the matrix with the rows $C_{n}^{[m]}=0$ for $0 \leq n \leq m$ and $C_{n}^{[m]}=C_{n}$ for $n \geq m+1$. It follows from (2.1), Lemma 3.2, (2.3), (3.4), 
and (3.2) that

$$
\begin{aligned}
\|L\|_{\chi_{\ell_{p}(\widehat{F})}} & \chi_{\ell_{p}(\widehat{F})}(L(S))=\chi_{\ell_{p}}\left(L_{C}(S)\right)=\lim _{m \rightarrow \infty}\left(\sup _{x \in S}\left\|\mathcal{R}_{m}(C x)\right\|_{p}\right) \\
& =\lim _{m \rightarrow \infty}\left(\sup _{x \in S}\left\|C^{[m]} x\right\|_{p}\right)=\lim _{m \rightarrow \infty}\left\|C^{[m]}\right\| \\
& =\lim _{m \rightarrow \infty}\left(\sup _{k} \sum_{n=m+1}^{\infty}\left|\frac{f_{n}}{f_{n+1}} a_{j k}-\frac{f_{n+1}}{f_{n}} a_{j-1, k}\right|^{p}\right)^{1 / p} .
\end{aligned}
$$

This completes the proof.

Finally, the characterization of $\mathcal{C}\left(\ell_{1}, \ell_{p}(\widehat{F})\right)$ is an immediate consequence of Theorem 3.2 and (2.2).

Theorem 3.3 Let $L \in \mathcal{B}\left(\ell_{1}, \ell_{p}(\widehat{F})\right)(1 \leq p<\infty)$ and A denote the matrix which represents $L$. Then $L$ is compact if and only if

$$
\lim _{m \rightarrow \infty}\left(\sup _{k} \sum_{n=m}^{\infty}\left|\frac{f_{n}}{f_{n+1}} a_{j k}-\frac{f_{n+1}}{f_{n}} a_{j-1, k}\right|^{p}\right)=0 .
$$

\section{Conclusions}

The Hausdorff measure of noncompactness can be most effectively used to characterize compact operators between Banach spaces. We have shown how the Hausdorff measure of noncompactness could be applied in the characterization of compact matrix operators between $B K$ spaces. Here we characterized the classes $\mathcal{B}\left(\ell_{1}, \ell_{p}(\widehat{F})\right)$ for $(1 \leq p<\infty)$ and computed the norm of operators in $\mathcal{B}\left(\ell_{1}, \ell_{p}(\widehat{F})\right)$. We also determined the Hausdorff measure of noncompactness of operators in $\mathcal{B}\left(\ell_{1}, \ell_{p}(\widehat{F})\right)$ and then characterized the classes $\mathcal{C}\left(\ell_{1}, \ell_{p}(\widehat{F})\right)$ for $1 \leq p<\infty$.

\section{Competing interests}

The authors declare that they have no competing interests.

\section{Authors' contributions}

The authors contributed equally and significantly in writing this paper. All authors read and approved the final manuscript.

\section{Acknowledgements}

This project was funded by the Deanship of Scientific Research (DSR) at King Abdulaziz University, Jeddah, under grant no. (69-130-35-RG). The authors, therefore, acknowledge with thanks DSR technical and financial support.

Received: 19 March 2015 Accepted: 22 May 2015 Published online: 18 June 2015

\section{References}

1. Basar, F: Summability Theory and Its Applications. Bentham Science Publishers, Istanbul (2011)

2. Wilansky, A: Summability Through Functional Analysis. North-Holland Mathematics Studies, vol. 85. Elsevier, Amsterdam (1984)

3. Banaś, J, Mursaleen, M: Sequence Spaces and Measures of Noncompactness with Applications to Differential and Integral Equations. Springer, Berlin (2014)

4. Koshy, T: Fibonacci and Lucas Numbers with Applications. Wiley, New York (2001)

5. Başarır, M, Başar, F, Kara, EE: On the spaces of Fibonacci difference null and convergent sequences (2013). arXiv:1309.0150v1 [math.FA]

6. Kara, EE: Some topological and geometrical properties of new Banach sequence spaces. J. Inequal. Appl. 2013, 38 (2013)

7. Kuratowski, K: Sur les espaces complets. Fundam. Math. 15, 301-309 (1930)

8. Darbo, G: Punti uniti in transformazioni a condominio non compatto. Rend. Semin. Mat. Univ. Padova 24, 84-92 (1955) 
9. Goldenštein, LS, Gohberg, IT, Markus, AS: Investigations of some properties of bounded linear operators with their q-norms. Učen. Zap. Kishinevsk. Univ. 29, 29-36 (1957)

10. Goldenštein, LS, Markus, AS: On a measure of noncompactness of bounded sets and linear operators. In: Studies in Algebra and Mathematical Analysis, pp. 45-54. Karta Moldovenjaske, Kishinev (1965)

11. Akhmerov, RR, Kamenskij, MI, Potapov, AS, Rodkina, AE, Sadovskii, BN: Measures of Noncompactness and Condensing Operators. Operator Theory: Advances and Applications, vol. 55. Birkhäuser, Basel (1992)

12. Ayerbe Toledano, JM, Domínguez Benavides, T, López Azedo, G: Measures of Noncompactness in Metric Fixed Point Theory. Birkhäuser, Basel (1997)

13. Banaś, J, Goebel, K: Measures of Noncompactness in Banach Spaces. Lecture Notes in Pure and Applied Mathematics, vol. 60. Dekker, New York (1980)

14. Malkowsky, E, Rakočević, V: An introduction into the theory of sequence spaces and measures of noncompactness. Zb. Rad. (Beogr.) 9(17), 143-234 (2000)

15. Alotaibi, A, Malkowsky, E, Mursaleen, M: Measure of noncompactness for compact matrix operators on some $B K$ spaces. Filomat 28, 1081-1086 (2014)

16. Başarı, M, Kara, EE: On compact operators on the Riesz $B^{(m)}$-difference sequence spaces. Iran. J. Sci. Technol., Trans. A, Sci. 35(A4), 279-285 (2011)

17. Başarır, M, Kara, EE: On some difference sequence spaces of weighted means and compact operators. Ann. Funct. Anal. 2, 114-129 (2011)

18. Başarır, M, Kara, EE: On the $B$-difference sequence space derived by generalized weighted mean and compact operators. J. Math. Anal. Appl. 391, 67-81 (2012)

19. Kara, EE, Başarır, M: On compact operators and some Euler B(m)-difference sequence spaces. J. Math. Anal. Appl. 379, 499-511 (2011)

20. de Malafosse, B, Malkowsky, E, Rakočević, V: Measure of noncompactness of operators and matrices on the spaces C and $c_{0}$. Int. J. Math. Math. Sci. 2006, 1-5 (2006)

21. de Malafosse, B, Rakočević, V: Applications of measure of noncompactness in operators on the spaces $s_{\alpha}, s_{\alpha}^{0}, s_{\alpha}^{(c)}, \ell_{\alpha}^{p}$. J. Math. Anal. Appl. 323(1), 131-145 (2006)

22. Mursaleen, M, Karakaya, V, Polat, H, Simsek, N: Measure of noncompactness of matrix operators on some difference sequence spaces of weighted means. Comput. Math. Appl. 62, 814-820 (2011)

23. Mursaleen, M, Mohiuddine, SA: Applications of measures of noncompactness to the infinite system of differential equations in $\ell_{p}$ spaces. Nonlinear Anal. 75, 2111-2115 (2012)

24. Mursaleen, M, Noman, AK: Compactness by the Hausdorff measure of noncompactness. Nonlinear Anal. 73, 2541-2557 (2010)

25. Mursaleen, M, Noman, AK: Compactness of matrix operators on some new difference sequence spaces. Linear Algebra Appl. 436(1), 41-52 (2012)

26. Kara, EE, Başarır, M, Mursaleen, M: Compactness of matrix operators on some sequence spaces derived by Fibonacci numbers (2013). arXiv:1309.0152v1 [math.FA]

\section{Submit your manuscript to a SpringerOpen ${ }^{\circ}$ journal and benefit from:}

- Convenient online submission

Rigorous peer review

- Immediate publication on acceptance

- Open access: articles freely available online

- High visibility within the field

- Retaining the copyright to your article 